\title{
Les réseaux d'épidémiosurveillance des maladies animales en Afrique francophone de l'Ouest et du Centre
}

\author{
M. Ouagal (1), P. Hendrikx (2), D. Berkvens ${ }^{(3)}$, A. Ncharé ${ }^{(4)}$, B. Cissé ${ }^{(5)}$, \\ P.Y. Akpeli ${ }^{(6)}$, K. Sory ${ }^{(7)} \&$ C. Saegerman ${ }^{(8)}$ \\ (1) Ministère de l'Élevage, Direction des Services vétérinaires, Service d'Épidémiologie, \\ B.P. 750, N’Djaména, Tchad \\ (2) Service de coopération et d'action culturelle (SCAC), Ambassade de France, Épidémiosurveillance, \\ développement rural et environnement, B.P. 780, Saint-Domingue, République Dominicaine \\ (3) Institut de médecine tropicale Prince Léopold, Département de santé animale \\ Unité d'épidémiologie et de statistiques appliquées, Nationalestraat 155, B-2000 Anvers, Belgique \\ (4) Ministère de l'Élevage, des Pêches et des Industries animales, \\ Direction des Services vétérinaires, B.P. 2228, Yaoundé, Cameroun \\ (5) Ministère de la Production animale et des Ressources halieutiques, \\ Laboratoire central vétérinaire, B.P. 206, Bingerville, Côte d'Ivoire \\ (6) Ministère de l'Agriculture, de l'Élevage et de la Pêche, Direction de l'élevage \\ et de la pêche, B.P. 4041, Lomé, Togo \\ (7) Ministère de l'Agriculture et de l'Élevage, Direction nationale de l'élevage, B.P. 559, Conakry, Guinée \\ (8) Université de Liège, Faculté de médecine vétérinaire, Département des maladies infectieuses \\ et parasitaires, Unité de Recherche en épidémiologie et analyse de risques appliquées aux sciences \\ vétérinaires, Boulevard de Colonster 20, B42 Sart-Tilman, B 4000 Liège, Belgique
}

Date de soumission : 22 janvier 2008

Date d'acceptation : 30 juin 2008

\section{Résumé}

L'un des objectifs du Programme panafricain de contrôle des épizooties (PACE) était la mise en place de réseaux d'épidémiosurveillance dans les pays d'Afrique. Une enquête, sur la base d'un questionnaire écrit, visant à faire un état des lieux de l'organisation technique et institutionnelle de ces réseaux a été réalisée dans neuf pays francophones, dont cinq en Afrique de l'Ouest (Sénégal, Burkina Faso, Côte d'Ivoire, Togo, Guinée) et quatre en Afrique centrale (Cameroun, République centrafricaine, République démocratique du Congo et Tchad). Les résultats de cette enquête ont montré qu'il existe plus de similitudes que de divergences entre ces réseaux. D'une manière générale, ils étaient techniquement et institutionnellement bien formalisés. Néanmoins, l'insuffisante capacité de diagnostic des laboratoires ainsi que l'insuffisante opérationnalité des comités de pilotage constituent deux points faibles de la majorité des réseaux. La surveillance épidémiologique devrait être une activité régalienne des Services vétérinaires et elle est déterminante pour assurer la détection rapide d'une modification du statut sanitaire d'une population animale; cependant, la pérennité des réseaux n'est généralement pas garantie en fin de financement extérieur car, souvent, leur prise en charge n'est pas assurée convenablement par les États.

\section{Mots-clés}

Afrique centrale - Afrique de l'Ouest - Animal domestique - Laboratoire - Maladie animale - Programme panafricain de contrôle des épizooties (PACE) - Réseau d'épidémiosurveillance - Services vétérinaires - Surveillance épidémiologique. 


\section{Introduction}

Il est essentiel de disposer d'un réseau d'épidémiosurveillance des maladies animales et d'un système de déclaration des maladies animales prioritaires dans les pays où l'élevage occupe une place prépondérante dans l'économie (18). En Afrique, depuis 1999, grâce au Programme panafricain de contrôle des épizooties (PACE) dont l'un des objectifs principaux était la mise en place de réseaux d'épidémiosurveillance des maladies animales, l'on a assisté à l'émergence et au renforcement de ceux-ci. Le PACE a été mis en œuvre dans 30 pays d'Afrique de l'Ouest, du Centre et de l'Est (11).

Un réseau d'épidémiosurveillance est un outil de prévention et d'aide à la décision (22); par conséquent, il doit produire des informations pertinentes, de qualité et de quantité suffisantes et en temps réel. À ce jour, les principes généraux de la mise en place des réseaux d'épidémiosurveillance sont bien connus et codifiés dans le Code sanitaire pour les animaux terrestres de l'Organisation mondiale de la santé animale (OIE) (12) ainsi que dans des guides pratiques de l'Organisation des Nations unies pour l'alimentation et l'agriculture (FAO) (par exemple, 14). La qualité d'un réseau d'épidémiosurveillance dépend de son organisation technique et institutionnelle $(5,18)$. Une précédente évaluation semi-quantitative menée dans 13 pays d'Afrique de l'Ouest a permis de montrer que l'état de mise en œuvre des réseaux d'épidémiosurveillance dans ces pays n'était pas homogène (19). L'objectif de cet article est de dresser un état des lieux de l'organisation technique et institutionnelle de quelques réseaux existants en Afrique francophone de l'Ouest et du Centre afin d'en dégager les similitudes, les divergences, les points forts, les points faibles et de proposer des axes d'amélioration.

\section{Matériels et méthodes}

Pour atteindre l'objectif de cette étude, une enquête à base d'un questionnaire écrit a été réalisée en 2006. Ce questionnaire prend en compte toutes les étapes techniques et organisationnelles nécessaires à la mise en œuvre d'un réseau d'épidémiosurveillance. Il a été envoyé par courrier électronique aux animateurs de réseaux d'épidémiosurveillance de 13 pays d'Afrique francophone dont neuf en Afrique de l'Ouest et quatre en Afrique Centrale. Les pays concernés étaient le Cameroun, la République centrafricaine (RCA), la République démocratique du Congo (RDC) et le Tchad pour l'Afrique du Centre ; le Sénégal, le Burkina Faso, la Côte d'Ivoire, le Togo, la Guinée (Conakry), le Niger, le Mali, la Mauritanie et le Bénin pour l'Afrique de l'Ouest. Les réponses et certaines informations complémentaires ou manquantes ont été obtenues de la même manière ainsi qu'à travers des entretiens téléphoniques personnalisés. Des rapports de missions d'évaluation et d'audit réalisées dans ces pays, dans le cadre du PACE, ainsi que des revues bibliographiques ont également été consultés.

\section{Résultats}

Au total 9 pays sur 13 ont répondu au questionnaire. Le Bénin, le Mali, la Mauritanie et le Niger n'ont pas répondu au questionnaire d'enquête.

\section{Organisation institutionnelle}

Le réseau d'épidémiosurveillance le plus anciennement créé est celui du Tchad (7); viennent ensuite, ceux de la Guinée, du Sénégal, de la RCA et du Burkina Faso. Ces réseaux ont été créés avant le démarrage du PACE. Presque tous les réseaux d'épidémiosurveillance enquêtés sont sous la tutelle de la Direction des Services vétérinaires (DSV) à part celui de la Guinée qui dépend du Laboratoire central vétérinaire de diagnostic (Tableau I).

\section{Tableau I}

Année de création et pouvoir de tutelle des réseaux d'épidémiosurveillance en Afrique francophone de l'Ouest et du Centre ayant répondu à l'enquête

\begin{tabular}{lcc}
\hline Réseau d'épidémiosurveillance & Date de création & Tutelle \\
\hline Burkina Faso & 1999 & DSV \\
Cameroun & 2004 & DSV \\
Côte d'Ivoire & 2000 & DSV \\
Guinée & 1996 & LCVD \\
République centrafricaine & 1999 & DSV \\
République démocratique du Congo & 2003 & DSV \\
Sénégal & 1997 & DSV \\
Tchad & 1995 & DSV \\
Togo & 2003 & DSV \\
\hline
\end{tabular}

DSV : Direction des Services vétérinaires

LCVD : Laboratoire central vétérinaire de diagnostic

La majorité des réseaux d'épidémiosurveillance enquêtés ont été mis en place sur une base de financement de projets et plus particulièrement dans le cadre du PACE (Tableau II). Dans tous les réseaux enquêtés, les acteurs de terrain impliqués sont rémunérés pour leurs activités de surveillance épidémiologique en plus de leur salaire normal. On distingue deux types de rémunération des acteurs du réseau d'épidémiosurveillance : des indemnités à l'acte (c'est le cas en RDC, au Tchad et au Togo) et des indemnités forfaitaires dans les autres pays (Tableau II).

Dans l'organisation institutionnelle des réseaux d'épidémiosurveillance enquêtés, on note principalement 


\section{Tableau II}

Source de financement et type de rémunération des acteurs des réseaux d'épidémiosurveillance en Afrique francophone de I'Ouest et du Centre ayant répondu à l'enquête

\begin{tabular}{lcc}
\hline $\begin{array}{l}\text { Réseau } \\
\text { d'épidémiosurveillance }\end{array}$ & $\begin{array}{c}\text { Source } \\
\text { de financement }\end{array}$ & $\begin{array}{c}\text { Type de } \\
\text { rémunération } \\
\text { des acteurs }\end{array}$ \\
\hline Burkina Faso & PACE & Forfait \\
Cameroun & PACE & Forfait \\
Côte d'lvoire & PACE & Forfait \\
Guinée & PARC & Forfait \\
République centrafricaine & PARC & Acte \\
République démocratique du Congo & PACE & Forfait \\
Sénégal & - & Forfait \\
Tchad & PMDR & Acte \\
Togo & PACE & Acte \\
\hline
\end{tabular}

PACE : Programme panafricain de contrôle des épizooties (financé par I'Union européenne et coordonné par le Bureau interafricain des ressources animales de l'Union africaine [UA-IBAR])

PARC : Campagne panafricaine de lutte contre la peste bovine (Pan African Rinderpest Campaign)

PMDR : Projet multisectoriel de développement rural (financé par le Fonds d'aide de coopération française)

l'existence de cinq grandes structures, à savoir le comité de pilotage, le comité technique, les unités régionales, l'unité centrale et l'équipe mobile (Tableau III). On rencontre au Togo une «cellule d'exécution permanente » qui est l'équivalent d'une unité centrale.

Des réunions du comité de pilotage, du comité technique et de la cellule d'animation sont prévues dans tous les réseaux d'épidémiosurveillance. Notons cependant que dans tous les réseaux d'épidémiosurveillance, le comité de pilotage ne se réunit que rarement.

\section{Organisation technique}

\section{Les objectifs}

Les objectifs poursuivis par les réseaux d'épidémiosurveillance enquêtés sont très variés (Tableau IV). Cependant, une analyse plus fine montre qu'ils peuvent être résumés en deux objectifs essentiels de l'épidémiosurveillance qui sont :

- la détection des maladies nouvelles ou exotiques (épidémiovigilance), et

- la connaissance de l'importance en termes de prévalence, d'incidence et de répartition géographique et temporelle des maladies existantes (épidémiosurveillance).

\section{Les maladies surveillées}

Les maladies surveillées diffèrent d'un réseau d'épidémiosurveillance à un autre. Leur nombre varie de 5 à 12 (Tableau V). On note cependant que la péripneumonie contagieuse bovine (PPCB), la peste bovine, la peste des petits ruminants, la fièvre aphteuse et l'influenza aviaire sont les maladies communes à presque tous les réseaux. Viennent ensuite la peste porcine africaine et la maladie de Newcastle qui sont surveillées par plus de la moitié des pays.

\section{Échantillonnage}

Les postes vétérinaires des pays étudiés qui constituent la structure de base des Services vétérinaires sur le terrain, ne sont pas tous impliqués dans la surveillance épidémiologique. Le taux d'implication de ces postes dans cette surveillance varie de $7 \%$ à $91 \%$. La superficie couverte par un poste de surveillance (poste vétérinaire impliqué dans le réseau d'épidémiosurveillance) varie de $1132 \mathrm{~km}^{2}$ à $86815 \mathrm{~km}^{2}$ (Tableau VI).

Tableau III

Principales structures des réseaux d'épidémiosurveillance en Afrique francophone de l'Ouest et du Centre ayant répondu à l'enquête

\begin{tabular}{|c|c|c|c|c|c|}
\hline $\begin{array}{l}\text { Réseau } \\
\text { d'épidémiosurveillance }\end{array}$ & \multicolumn{5}{|c|}{ Éléments structurels } \\
\hline Burkina Faso & + & + & + & + & + \\
\hline Cameroun & + & + & + & + & + \\
\hline Côte d'Ivoire & + & + & + & + & + \\
\hline Guinée & + & + & + & + & + \\
\hline République centrafricaine & + & + & + & + & + \\
\hline République démocratique du Congo & + & + & + & - & + \\
\hline Sénégal & + & + & + & - & + \\
\hline Tchad & + & + & + & + & - \\
\hline Togo & + & + & $(+)$ & + & - \\
\hline
\end{tabular}

t: Élément existant

- : Élément inexistant

$(+)$ : Cellule d'exécution permanente (assimilée à une unité centrale) 
Tableau IV

Objectifs des réseaux d'épidémiosurveillance en Afrique francophone de l'Ouest et du Centre ayant répondu à l'enquête

\begin{tabular}{|c|c|}
\hline Pays enquêtés & Objectifs du réseau \\
\hline Burkina Faso & $\begin{array}{l}\text { - Détecter précocement et réagir rapidement en cas d'apparition, de réapparition ou d'introduction d'une nouvelle maladie dans le pays } \\
\text { - Mener des enquêtes pour déterminer la prévalence et l'incidence des maladies jugées prioritaires } \\
\text { - Fournir des informations nécessaires à la déclaration des maladies animales en respect des règles internationales } \\
\text { - Fournir des informations pour la prise de mesures appropriées }\end{array}$ \\
\hline Cameroun & $\begin{array}{l}\text { - Surveillance active de la peste bovine } \\
\text { - Surveillance épidémiologique des autres maladies prioritaires }\end{array}$ \\
\hline Côte d'Ivoire & $\begin{array}{l}\text { - Détection rapide et maîtrise des foyers des épizooties sur toute l'étendue du territoire national } \\
\text { - Détecter toute introduction de maladie nouvelle } \\
\text { - Déterminer l'importance réelle des maladies mises sous surveillance } \\
\text { - Mettre à la disposition des décideurs des informations épidémiologiques (aide à la décision) }\end{array}$ \\
\hline Guinée & - Surveillance permanente des maladies d'intérêt zoosanitaire et économique pour une détection précoce en vue d'une réaction rapide \\
\hline $\begin{array}{l}\text { République } \\
\text { centrafricaine }\end{array}$ & $\begin{array}{l}\text { - Détecter le plus rapidement possible toute suspicion de peste bovine sur le territoire national afin d'agir } \\
\text { le plus rapidement possible et éviter toute propagation de la maladie dans le pays } \\
\text { - Détecter le plus rapidement possible toute suspicion de péripneumonie contagieuse bovine (PPCB) sur le territoire national } \\
\text { - Connaître la situation sanitaire d'autres pathologies majeures dans les postes d'observation et en particulier } \\
\text { les maladies pestiformes devant être différenciées des cas de peste bovine }\end{array}$ \\
\hline $\begin{array}{l}\text { République } \\
\text { démocratique } \\
\text { du Congo }\end{array}$ & $\begin{array}{l}\text { - Enquête épidémiologique } \\
\text { - Surveillance des maladies prioritaires } \\
\text { - Déclenchement des alertes } \\
\text { - Mise à disposition des données utiles pour les plans de lutte contre les maladies surveillées }\end{array}$ \\
\hline Sénégal & $\begin{array}{l}\text { - La détection de toute résurgence et /ou réintroduction de maladie éradiquée au Sénégal et sa mise sous surveillance } \\
\text { - La détection de toute introduction nouvelle de maladies } \\
\text { - La confirmation de l'éradication de maladies } \\
\text { - L'évaluation des résultats des plans de lutte contre les maladies animales } \\
\text { - La détermination de l'importance réelle des maladies mises sous surveillance en étudiant leur incidence, leur prévalence } \\
\text { et leur importance économique } \\
\text { - La mise à la disposition de la Direction de l'élevage des informations épidémiologiques devant l'aider à prendre une décision } \\
\text { en matière de lutte contre les maladies animales } \\
\text { - L'adaptation au nouveau contexte de mondialisation du commerce et d'analyse des risques liés aux échanges } \\
\text { d'animaux et de produits d'origine animale }\end{array}$ \\
\hline Tchad & $\begin{array}{l}\text { - Surveiller les maladies animales jugées prioritaires } \\
\text { - Détecter précocement les nouveaux foyers de ces maladies } \\
\text { - Recueillir les informations concernant ces maladies en termes de répartition géographique et temporelle, de prévalence et d'incidence } \\
\text { - Transmettre ces informations aux autorités chargées de l'élevage, de la santé animale, et de la santé humaine } \\
\quad \text { pour ce qui concerne les maladies à caractère zoonotique }\end{array}$ \\
\hline Togo & $\begin{array}{l}\text { - Faire reconnaître le Togo indemne de peste bovine par l’Organisation mondiale de la santé animale (OIE) et mettre en place une veille } \\
\text { permanente consistant en une surveillance clinique, nécropsique bactériologique, virologique et sérologique pour prévenir une éventuelle } \\
\text { réapparition de la maladie } \\
\text { - Contrôle permanent des autres maladies de l'ancienne liste A de l’OIE }\end{array}$ \\
\hline
\end{tabular}

D’une manière générale, dans la plupart des pays enquêtés, les éleveurs, les auxiliaires d'élevage, les commerçants de bétail, les associations d'éleveurs, les agents des parcs nationaux sont impliqués dans le réseau d'épidémiosurveillance. En revanche, les vétérinaires privés, lorsqu'ils existent, ne sont pas tous ou pas du tout impliqués (Tableau VII).

\section{Collecte des données}

Plusieurs méthodes sont utilisées pour la collecte des données. Le plus souvent, une surveillance passive est pratiquée (6). En complément, une surveillance active, suivant la procédure de l'OIE (9), est pratiquée pour l'obtention du statut de pays indemne de peste bovine. 


\section{Tableau V}

Liste des maladies surveillées par les réseaux d'épidémiosurveillance en Afrique francophone de l'Ouest et du Centre ayant répondu à l'enquête

\begin{tabular}{|c|c|c|c|c|c|c|c|c|c|c|c|c|c|c|c|c|c|c|}
\hline \multirow{2}{*}{$\begin{array}{l}\text { Réseau } \\
\text { d'epidémiosurveillance }\end{array}$} & \multicolumn{17}{|c|}{ Maladies surveillées } & \multirow[t]{2}{*}{ Total } \\
\hline & PB & РРСВ & PPR & FA & IA & PPA & NC & DNB & FVR & TRY & TUB & CB & CS & B & PE & PPCC & $\mathbf{P}$ & \\
\hline Burkina Faso & $x$ & $x$ & $x$ & $X$ & $x$ & $X$ & & & & & & & & & & & & 6 \\
\hline Cameroun & $x$ & $x$ & $x$ & $X$ & $x$ & $X$ & $x$ & & & & & & & & & & & 7 \\
\hline Côte d'Ivoire & $x$ & $x$ & $x$ & $x$ & $x$ & $X$ & $x$ & $x$ & & & & & & & & & & 8 \\
\hline Guinée & $x$ & $x$ & $x$ & $x$ & $x$ & & & & & & & & & & & & & 5 \\
\hline République centrafricaine & $x$ & $x$ & $x$ & & & & & & & $x$ & & & & $x$ & & & & 5 \\
\hline $\begin{array}{l}\text { République démocratique } \\
\text { du Congo }\end{array}$ & $x$ & $x$ & $x$ & $x$ & $x$ & $x$ & & & & & & & & & & & & 6 \\
\hline Sénégal & $x$ & $x$ & $x$ & $x$ & $x$ & $x$ & $x$ & $x$ & $x$ & & & & & & $x$ & & & 10 \\
\hline Tchad & $x$ & $x$ & $x$ & $x$ & $x$ & & $x$ & & $x$ & & $x$ & $x$ & $x$ & & & $x$ & $x$ & 12 \\
\hline Togo & $x$ & $x$ & $x$ & $x$ & $x$ & $x$ & $x$ & $x$ & & $x$ & $x$ & $x$ & $x$ & & & & & 12 \\
\hline Total & 9 & 9 & 9 & 8 & 8 & 6 & 5 & 3 & 2 & 2 & 2 & 2 & 2 & 1 & 1 & 1 & 1 & 71 \\
\hline $\begin{array}{l}\text { B: } \text { babésiose } \\
\text { DNB : dermatose nodulaire conta } \\
\text { FA : fièvre aphteuse } \\
\text { FVR : fièvre de la Vallée du Rift } \\
\text { IA : influenza aviaire } \\
\text { NC: maladie de Newcastle } \\
\text { PB : peste bovine } \\
\text { PE: peste équine } \\
P: \text { pasteurellose }\end{array}$ & gieuse & Dovine & & & & & & & $\begin{array}{ll}\text { PPA }: & p \\
\text { PPCB }: & p \\
\text { PPR }: & p \\
\text { TRY : } & t \\
\text { PPCC }: & p \\
\text { TUB }: & t \\
\text { CB }: & f \\
C S: & c \\
X: & n\end{array}$ & $\begin{array}{l}\text { ste porci } \\
\text { sipneum } \\
\text { ste des } \\
\text { ypanoson } \\
\text { europnet } \\
\text { berculos } \\
\text { evre char } \\
\text { tarbon sy } \\
\text { aladie re }\end{array}$ & $\begin{array}{l}\text { ine africa } \\
\text { onie cont } \\
\text { petits run } \\
\text { nose } \\
\text { umonie c } \\
\text { e } \\
\text { bonneus } \\
\text { mptomat } \\
\text { tenue po }\end{array}$ & $\begin{array}{l}\text { aine } \\
\text { tagieuse } \\
\text { minants } \\
\text { ontagie } \\
\text { e } \\
\text { tique } \\
\text { ur la su }\end{array}$ & $\begin{array}{l}\text { Dovine } \\
\text { e caprine } \\
\text { eillance ép }\end{array}$ & & gique & & & \\
\hline
\end{tabular}

\section{Tableau VI}

Pourcentage de postes vétérinaires impliqués dans la surveillance épidémiologique et superficie couverte par chacun des postes en Afrique francophone de l'Ouest et du Centre (réseaux ayant répondu à l'enquête)

\begin{tabular}{|c|c|c|c|c|c|}
\hline Réseau d'épidémiosurveillance & $\begin{array}{c}\text { Superficie } \\
\text { du pays }\left(\mathbf{k m}^{2}\right)\end{array}$ & $\begin{array}{l}\text { Nombre de postes } \\
\text { vétérinaires (a) }\end{array}$ & $\begin{array}{l}\text { Nombre de postes } \\
\text { de surveillance }\end{array}$ & $\begin{array}{l}\text { Pourcentage de postes } \\
\text { vétérinaires impliqués } \\
\text { dans la surveillance }\end{array}$ & $\begin{array}{c}\text { Superficie couverte } \\
\text { par le poste de } \\
\text { surveillance }\left(\mathbf{k m}^{2}\right)\end{array}$ \\
\hline Burkina Faso & 274000 & 100 & 45 & 45 & 6089 \\
\hline Cameroun & 475000 & 700 & 52 & 7 & 9500 \\
\hline Côte d'Ivoire & 322000 & 83 & 32 & 85 & 10063 \\
\hline Guinée & 246000 & 340 & 31 & 85 & 7935 \\
\hline République centrafricaine & 623000 & 110 & 42 & 38 & 14833 \\
\hline République démocratique du Congo & 2344000 & 58 & 27 & 47 & 86815 \\
\hline Sénégal & 197000 & 145 & 49 & 34 & 4020 \\
\hline Tchad & 1284000 & 154 & 131 & 85 & 9802 \\
\hline Togo & 56600 & 55 & 50 & 91 & 1132 \\
\hline
\end{tabular}

(a) Structures de base des Services vétérinaires sur le terrain

(b) Postes vétérinaires impliqués dans un réseau d'épidémiosurveillance

Plusieurs pays effectuent également des visites régulières de villages (troupeaux) pour la recherche des maladies surveillées (surveillance active). C'est le cas du Burkina Faso, de la RCA, du Sénégal et du Tchad.

Tous les agents des postes de surveillance des réseaux d'épidémiosurveillance des pays enquêtés ont suivi une formation initiale et des sessions annuelles de recyclage. Les matériels de collecte des données (fiches, matériel de prélèvement, glacières) sont généralement disponibles dans les postes de surveillance. Pour les déplacements, les agents utilisent le plus souvent des motocyclettes. Le pourcentage de postes de surveillance qui disposent de motocyclettes varie de $34 \%$ 


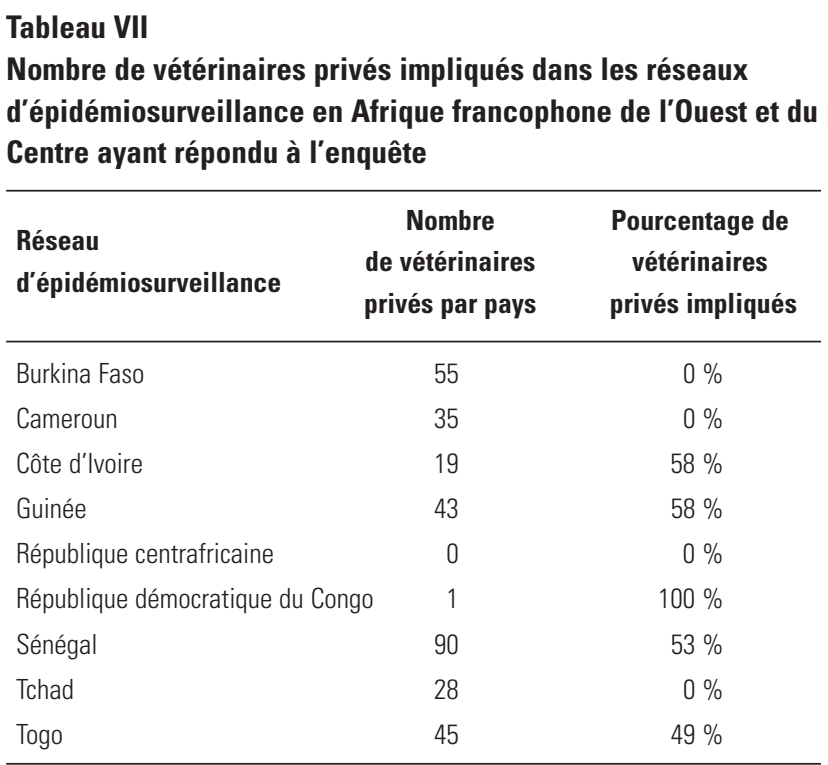

\section{Tableau VIII}

Pourcentage de postes de surveillance épidémiologique ayant des moyens de déplacement en Afrique francophone de l'Ouest et du Centre (réseaux d'épidémiosurveillance ayant répondu à I'enquête)

\begin{tabular}{lccc}
\hline Réseau & $\begin{array}{c}\text { Nombre } \\
\text { de poste } \\
\text { de surveillance }\end{array}$ & $\begin{array}{c}\text { Nombre de } \\
\text { motocyclettes } \\
\text { disponibles }\end{array}$ & $\begin{array}{c}\text { Pourcentage des } \\
\text { postes de surveillance } \\
\text { ayant des motocyclettes }\end{array}$ \\
\hline Burkina Faso & 45 & 45 & $100 \%$ \\
Côte d'Ivoire & 32 & 32 & $100 \%$ \\
Cameroun & 52 & 32 & $62 \%$ \\
Guinée & 31 & 31 & $100 \%$ \\
République & 42 & 28 & $67 \%$ \\
centrafricaine & & & $100 \%$ \\
République & 27 & 27 & \\
démocratique & & & $67 \%$ \\
du Congo & & 33 & $34 \%$ \\
Sénégal & 49 & 45 & $100 \%$ \\
Tchad & 131 & 50 & \\
Togo & 50 & &
\end{tabular}

à $100 \%$ (Tableau VIII). Certains postes qui ne disposent pas de motocyclettes utilisent leur propre moyen de déplacement et sont rémunérés en conséquence (Tchad).

\section{Transmission des données}

Comme le montre le Tableau IX, dans tous les réseaux d'épidémiosurveillance des pays enquêtés, les données collectées par les postes de surveillance transitent par une structure intermédiaire, généralement une unité régionale, avant d'arriver à l'unité centrale. La diffusion des résultats suit presque le même circuit en sens inverse.

\section{Gestion et traitement des données}

Tous les réseaux d'épidémiosurveillance des pays enquêtés possèdent pour la gestion des données, une base de données intégrée développée à l'échelon régional dans le cadre du programme PACE (Pace Integrated Database/Animal Ressources Information System, PID/ARIS). En plus de cette base, les pays comme la Guinée, la RDC, le Sénégal et le Tchad utilisent une base de données nationale écrite à l'aide du logiciel Access ${ }^{\circledR}$. Notons cependant que près de la moitié des pays enquêtés affirment avoir des problèmes dans l'utilisation du PID/ARIS (manque de flexibilité, blocage). Le traitement des données est généralement réalisé à l'aide des logiciels PID/ARIS, Excel ${ }^{\circledR}$ et Access $^{\circledR}$ (Tableau X).

Tous les pays enquêtés disposent d'un laboratoire national central pour les analyses des prélèvements collectés. Des laboratoires provinciaux existent également, sauf au Tchad, en RDC et au Togo. Ces laboratoires sont considérés comme des partenaires des réseaux d'épidémiosurveillance dans la plupart des pays enquêtés. Notons cependant que seuls quatre pays (Cameroun, Côte d'Ivoire, Guinée et Sénégal) peuvent réaliser un diagnostic de toutes les maladies retenues pour la surveillance (Tableau XI).

\section{Diffusion de l'information}

La diffusion de l'information produite par les réseaux d'épidémiosurveillance des pays enquêtés se fait en général par un bulletin épidémiologique. La fréquence de publication de ces bulletins est trimestrielle, sauf au Cameroun où elle est semestrielle (Tableau XII). On note que cette fréquence n'est jamais respectée vu le nombre de bulletins publiés par rapport aux prévisions. D'autres supports tels que les rapports, les annonces, les réunions, les plaquettes, la radio, la télévision, le téléphone, les dépliants et les messages électroniques sont également utilisés pour la diffusion des résultats et la communication des informations aux acteurs du réseau d'épidémiosurveillance et autres personnes intéressées.

\section{Évaluation}

Tous les réseaux d'épidémiosurveillance enquêtés disposent d'indicateurs de performance (8), même s'ils ne sont pas toujours utilisés. La majorité des réseaux des pays enquêtés ont bénéficié au moins d'une évaluation externe organisée dans le cadre du PACE. Au Sénégal, au Tchad et au Togo, les indicateurs de performance sont utilisés pour des évaluations internes des réseaux d'épidémiosurveillance. 


\section{Tableau IX \\ Mode de transmission des données épidémiologiques au sein des réseaux d'épidémiosurveillance en Afrique francophone de l'Ouest et du Centre ayant répondu à l'enquête}

\begin{tabular}{|c|c|}
\hline Réseau d'épidémiosurveillance & Mode de transmission des données \\
\hline Burkina Faso & $\begin{array}{l}\text { Les fiches d'enquête sont acheminées mensuellement à l'unité centrale, soit directement par les transporteurs routiers, } \\
\text { soit par la voie administrative. Les informations sont données lors des réunions trimestrielles } \\
\text { et en dehors de ces réunions par la voie administrative }\end{array}$ \\
\hline Cameroun & $\begin{array}{l}\text { Le chef d'unité régionale centralise les données et prélèvements des différents postes de surveillance et se charge } \\
\text { de leur transfert à l'unité centrale ou au laboratoire. Le retour d'information se fait par le même canal } \\
\text { ou directement de l'unité centrale au poste. Les moyens utilisés sont le téléphone, I'Internet, le fax et le courrier postal }\end{array}$ \\
\hline Côte d'Ivoire & $\begin{array}{l}\text { L'agent du poste de surveillance transmet les données à la Direction des Services vétérinaires (DSV) ou au Chef du réseau } \\
\text { via le Directeur départemental ; mais les prélèvements d'échantillons sont transmis directement au laboratoire } \\
\text { (le Chef du réseau en faisant partie). La DSV transmet à son tour les informations au Chef du réseau pour traitement. } \\
\text { Les résultats sont envoyés à la fois au DSV, au poste de surveillance et au propriétaire des animaux par le Chef du réseau }\end{array}$ \\
\hline Guinée & $\begin{array}{l}\text { Les fiches d'enquêtes sont acheminées mensuellement par les agents des postes d'élevage vers les Sections préfectorales } \\
\text { des ressources animales des chefs-lieux qui les transmettent au responsable du réseau (LCVD). Les missions de supervision } \\
\text { sont également mises à profit pour rassembler les fiches d'enquêtes et le matériel biologique. Les résultats des analyses } \\
\text { sont transmis à la mission des Services vétérinaires qui les communique aux services du terrain et aux éleveurs. } \\
\text { Les résultats alimentent les informations communiquées à l'OIE et au Bureau interafricain des ressources animales } \\
\text { de I'Union africaine (UA-IBAR) }\end{array}$ \\
\hline République centrafricaine & $\begin{array}{l}\text { Les rapports mensuels du réseau sont exploités par le Chef de Service régional de santé animale (CSRSA) puis transmis } \\
\text { par le Directeur régional à la Coordination du programme PACE. Les observations sont renvoyées au CSRSA qui retrouve } \\
\text { l'agent du réseau pour lui en faire part. Les prélèvements et les rapports de suspicion de maladies infectieuses } \\
\text { ne suivent pas cette procédure. Ils peuvent être acheminés directement à la Coordination par le moyen le plus rapide. } \\
\text { En cas de suspicion de maladie contagieuse une copie du rapport est obligatoirement adressée par l'agent au Directeur } \\
\text { régional. Les résultats des examens de laboratoires sont diffusés à la radio nationale puis les fiches sont renvoyées } \\
\text { à l'agent du réseau qui en informe le propriétaire du troupeau }\end{array}$ \\
\hline République démocratique du Congo & $\begin{array}{l}\text { Les informations provenant des éleveurs, transitent par des agents de relais puis par des agents des postes } \\
\text { d'observation pour arriver à la cellule de coordination nationale via l'alimentation d'une banque des données. } \\
\text { Le retour de l'information se fait par l'édition d'un bulletin épidémiologique et des annonces diverses }\end{array}$ \\
\hline Sénégal & $\begin{array}{l}\text { L'acheminement des fiches de collecte et des prélèvements se fait par les taxis brousse (prise en charge } \\
\text { par le programme PACE). Les agents reçoivent une indemnité forfaitaire si les prélèvements sont de qualité } \\
\text { et les fiches bien renseignées. Le retour de l'information se fait par l'envoi de lettres contenant les résultats } \\
\text { de laboratoire (sont disponibles aussi sur un réseau intranet) et l'édition d'un bulletin épidémiologique }\end{array}$ \\
\hline Tchad & $\begin{array}{l}\text { Les données collectées par les agents de surveillance sont transmises au chef de secteur qui les achemine } \\
\text { à la cellule d'animation. Les résultats sont transmis soit directement au poste de surveillance par la cellule d'animation, } \\
\text { qui à son tour les transmet aux éleveurs, soit indirectement au chef de secteur, qui se charge de les communiquer } \\
\text { au poste de surveillance }\end{array}$ \\
\hline Togo & Les données de terrain sont transmises aux unités régionales qui les adressent à l'unité centrale \\
\hline
\end{tabular}

\section{Discussion}

Le PACE a été un programme important pour les États d'Afrique qui y ont pris part. Il a permis à la plupart de ces pays de mettre en place un réseau d'épidémiosurveillance dont l'utilité et la nécessité à l'heure de la mondialisation ne sont plus à démontrer. Cette étude a montré qu'il existe plus de similitudes que de divergences entre les neuf réseaux d'épidémiosurveillance enquêtés. Ceci est dû au fait qu'une coordination régionale, maintenue grâce au programme PACE, a diffusé un discours commun sur l'organisation des réseaux d'épidémiosurveillance. De la même manière, cette approche pourrait être suivie pour :

- harmoniser les procédures et les protocoles de surveillance épidémiologique entre ces pays comme cela se fait déjà, par exemple, pour la grippe aviaire,

- bâtir des outils d'évaluation et de suivi des réseaux d'épidémiosurveillance à l'échelon régional comme cela se fait aux Caraïbes (2). 
Tableau X

Logiciels de base de données et de traitement des données utilisés par les réseaux d'épidémiosurveillance en Afrique francophone de l'Ouest et du Centre ayant répondu à l'enquête

\begin{tabular}{|c|c|c|c|c|c|c|c|c|}
\hline \multirow{2}{*}{$\begin{array}{l}\text { Réseau } \\
\text { d'd'épidémiosurveillance }\end{array}$} & \multicolumn{2}{|c|}{ Logiciel de base de données } & \multicolumn{6}{|c|}{ Logiciel de traitement des données } \\
\hline & PID/ARIS ${ }^{\text {(a) }}$ & Access & PID/ARIS & Excel & Access & SID 3.0 & Arcview & Stata \\
\hline Burkina Faso & $X$ & & & $x$ & & & & \\
\hline Cameroun & $x$ & & $x$ & & & & & \\
\hline Côte d'Ivoire & $x$ & & $x$ & $x$ & & & & \\
\hline Guinée & $x$ & $x$ & $x$ & $x$ & & $x$ & & \\
\hline République centrafricaine & $x$ & & $x$ & $x$ & & & & \\
\hline République démocratique du Congo & $x$ & $x$ & $x$ & & $x$ & & & \\
\hline Sénégal & $x$ & $x$ & $x$ & & $x$ & & $x$ & \\
\hline Tchad & $x$ & $x$ & $x$ & $x$ & $x$ & & & $x$ \\
\hline Togo & $x$ & & $x$ & & & & & \\
\hline Total & 9 & 4 & 8 & 5 & 3 & 1 & 1 & 1 \\
\hline
\end{tabular}

(a) Base de données intégrée développée à l'échelon régional dans le cadre du Programme panafricain de contrôle des épizooties (PACE) (Pace Integrated Database/Animal Ressources Information System, PID/ARIS)

\section{Tableau XI}

Nombre de maladies analysables par les laboratoires nationaux des réseaux d'épidémiosurveillance en Afrique francophone de I'Ouest et du Centre ayant répondu à l'enquête

\begin{tabular}{|c|c|c|c|}
\hline $\begin{array}{l}\text { Réseau } \\
\text { d'épidémiosurveillance }\end{array}$ & $\begin{array}{l}\text { Nombre de maladies } \\
\text { retenues pour la surveillance }\end{array}$ & $\begin{array}{c}\text { Nombre de maladies analysables } \\
\text { par les laboratoires nationaux }\end{array}$ & $\begin{array}{l}\text { Type de maladies analysables } \\
\text { par les laboratoires nationaux }\end{array}$ \\
\hline Burkina Faso & 6 & 3 & PB, PPR, PPCB \\
\hline Cameroun & 7 & 7 & Tous \\
\hline Côte d'Ivoire & 8 & 8 & Tous \\
\hline Guinée & 5 & 5 & Tous \\
\hline République centrafricaine & 5 & 2 & TRY, B \\
\hline République démocratique du Congo & 6 & 2 & $\mathrm{~PB}, \mathrm{FA}$ \\
\hline Sénégal & 10 & 10 & Tous \\
\hline Tchad & 12 & 7 & PB, PPR \\
\hline Togo & 12 & 1 & PB \\
\hline
\end{tabular}

\begin{tabular}{ll}
\hline B : babésiose & PPCB : péripneumonie contagieuse bovine \\
FA : fièvre aphteuse & PPR : peste des petits ruminants \\
PB : peste bovine & TRY: trypanosomose
\end{tabular}

Tableau XII

Fréquence de publication des bulletins des réseaux d'épidémiosurveillance de l'Afrique francophone de l'Ouest et du Centre ayant répondu à l'enquête (depuis leur création en 2006)

\begin{tabular}{|c|c|c|c|c|}
\hline Pays enquêtés & $\begin{array}{l}\text { Fréquence } \\
\text { du bulletin }\end{array}$ & $\begin{array}{c}\text { Nombre de } \\
\text { bulletins prévus }\end{array}$ & $\begin{array}{c}\text { Nombre de } \\
\text { bulletins publiés }\end{array}$ & $\begin{array}{c}\text { Pourcentage de réalisation } \\
\text { des bulletins }\end{array}$ \\
\hline Burkina Faso & Trimestrielle & 24 & 1 & $4 \%$ \\
\hline Cameroun & Semestrielle & 4 & 2 & $50 \%$ \\
\hline Côte d'Ivoire & Trimestrielle & 24 & 0 & $0 \%$ \\
\hline Guinée & Trimestrielle & 36 & 4 & $11 \%$ \\
\hline République centrafricaine & Trimestrielle & 24 & 18 & $75 \%$ \\
\hline République démocratique du Congo & Trimestrielle & 24 & 4 & $17 \%$ \\
\hline Sénégal & Trimestrielle & 36 & 18 & $50 \%$ \\
\hline Tchad & Trimestrielle & 40 & 20 & $50 \%$ \\
\hline Togo & Trimestrielle & 12 & 4 & $33 \%$ \\
\hline
\end{tabular}


La tutelle des réseaux d'épidémiosurveillance des maladies animales a, dans les premières années de création, entraîné des débats. Ce fut le cas du réseau d'épidémiosurveillance des maladies animales au Tchad (REPIMAT) qui, à sa création, était sous la tutelle d'un laboratoire (8) avant d'être finalement placé sous celle de la Direction des Services vétérinaires en 2001, au démarrage du PACE (13). Un réseau d'épidémiosurveillance est un outil de prévention et d'aide à la décision au bénéfice des Services vétérinaires, le laboratoire restant un partenaire incontournable et pas seulement un prestataire de services. En inadéquation avec le caractère panafricain du PACE, le réseau d'épidémiosurveillance est considéré comme une structure indépendante dans certains États africains. Dans ce contexte, sa pérennité est alors mise en jeu. De par sa nature, la surveillance épidémiologique est une activité régalienne des Services vétérinaires et un réseau d'épidémiosurveillance efficace s'avère déterminant pour assurer la détection rapide d'une modification du statut sanitaire d'une population animale (17). Le maintien des réseaux d'épidémiosurveillance au sein des Services vétérinaires est donc un des éléments permettant d'en assurer la légitimité et la pérennité, pour peu que la question de l'organisation entre les institutions partenaires ait été abordée efficacement et sereinement.

Généralement, un réseau d'épidémiosurveillance implique plusieurs structures ou organismes. Pour assurer son bon fonctionnement, la formalisation d'organes de coordination et de régulation s'impose. Cette étude a montré que tous les réseaux d'épidémiosurveillance enquêtés disposent de structures de collaboration et d'animation bien formalisées mais que ces structures ne sont pas souvent fonctionnelles, surtout le comité de pilotage qui est une instance de prise de décision politique et d'orientation du réseau d'épidémiosurveillance, car il ne se réunit que rarement. Les membres des comités de pilotage étaient souvent des hauts fonctionnaires issus de différentes administrations, ce qui peut expliquer la difficulté d'organiser régulièrement des réunions de ces comités.

L'enquête a montré également que presque tous les réseaux d'épidémiosurveillance étudiés ont été mis en place par des projets ou programmes, et leur fonctionnement est assuré pour l'essentiel par des financements extérieurs. En tant qu'activité pérenne, un réseau d'épidémiosurveillance ne peut être exclusivement supporté par des projets ou programmes dont la durée est limitée dans le temps. Par conséquent, la prise en charge du fonctionnement des réseaux d'épidémiosurveillance par les États est une nécessité. Certes, certains États connaissent des tensions de trésorerie qui font que les ressources sont d'abord affectées à des secteurs jugés prioritaires comme la santé humaine ou l'éducation. Mais il faut noter que le coût du fonctionnement d'un réseau d'épidémiosurveillance est nettement moins important que les conséquences de l'introduction d'une maladie ou la mise en ouvre d'un programme de contrôle ou d'éradication de celle-ci $(20,21)$. Cet engagement de l'État devrait être sollicité par le comité de pilotage, car c'est un de ses rôles d'assurer et de rechercher le financement du réseau. Le fonctionnement d'un réseau d'épidémiosurveillance nécessite un minimum de moyens parmi lesquels le moyen de déplacement, sans lequel il serait difficile, voire impossible, pour un agent d'un poste de surveillance d'investiguer les suspicions éloignées de sa zone de résidence. Par conséquent, il est indispensable que tous les postes de surveillance disposent au moins d'un moyen de déplacement.

La motivation des acteurs du réseau est un point essentiel mais, comme signalé auparavant, l'activité de surveillance épidémiologique fait partie intégrante des tâches usuelles des Services vétérinaires sur le terrain pour lesquelles les agents perçoivent un salaire. Les indemnités ou primes qui peuvent être allouées ne doivent donc pas être considérées comme un droit absolu. En fonction de disponibilités budgétaires, elles peuvent toutefois servir de moyens de motivation supplémentaires des agents. Dans ce cas, il est préférable d'allouer des indemnités à l'acte plutôt que d'attribuer des forfaits. Ce procédé permet de récompenser davantage les agents qui participent le plus aux activités du réseau d'épidémiosurveillance.

L'analyse des objectifs des réseaux d'épidémiosurveillance des pays enquêtés montre que ce sont tous des réseaux mixtes qui réalisent à la fois des activités d'épidémiosurveillance et d'épidémiovigilance et qui surveillent plusieurs maladies. Il n'existe presque pas de réseaux d'épidémiosurveillance spécifiques par espèce animale ou par maladie comme ceux rencontrés, par exemple, en France (4) ou en Belgique (15). Seul le Sénégal fait exception à cette règle, puisqu'il existe le Réseau sénégalais de surveillance des maladies des volailles (RESESAV) dédié spécifiquement à la surveillance des maladies des volailles (1). L'intérêt d'avoir un réseau national unique est sa capacité de s'adapter assez facilement à une modification telle que l'ajout d'une nouvelle maladie (3), comme ce fut le cas avec l'influenza aviaire qui a été intégrée par tous les réseaux d'épidémiosurveillance dès son apparition. L'objectif d'un réseau doit être clair, précis et tenir compte des ressources disponibles pour atteindre les résultats escomptés. Il ne sert à rien de fixer des objectifs si l'on sait d'avance que l'on n'a pas les moyens techniques, humains et financiers suffisants pour les atteindre. L'objectif de cette étude n'étant pas d'évaluer l'efficacité des réseaux mis en place mais uniquement leurs structures techniques et organisationnelles, il n'a pas été possible de faire une analyse critique des résultats de surveillance corrélés au nombre de maladies surveillées. 
La liste des maladies à surveiller est variable d'un pays à un autre. On constate néanmoins des maladies retenues communes, qui sont en général des maladies transfrontalières avec une importance économique et épidémiologique majeure (par exemple, l'influenza aviaire ou la PPCB). Le choix des maladies à surveiller dépend de la situation épidémiologique du pays et de l'importance accordée à ces maladies. Il n'est ni techniquement, ni économiquement possible de surveiller toutes les maladies existantes dans un pays. Des priorités doivent être fixées. Toutefois, des dispositions techniques doivent être mises en ouvre dans les protocoles de surveillance pour collecter les informations sur les suspicions des autres maladies préoccupantes qui ne font pas partie de la liste des maladies sous surveillance. Ceci est essentiel pour se donner les moyens de détecter l'apparition dans le pays d'une maladie animale nouvelle (encore inconnue ou exotique). Ces données peuvent également permettre de mieux apprécier les dominantes pathologiques à l'échelon national. Notons que depuis 2006, il n'existe plus qu'une liste unique de maladies notifiables à l'OIE (10). Ceci est important car cela donne plus de latitude à chaque pays pour établir un ordre de priorité des maladies qu'il souhaite surveiller.

Les laboratoires constituent la cheville ouvrière des programmes de surveillance mis en œuvre par les Services vétérinaires. Généralement, le fonctionnement d'un laboratoire nécessite des investissements conséquents. L'incapacité des laboratoires des pays enquêtés à traiter toutes les suspicions des maladies retenues pour la surveillance constitue un handicap au bon fonctionnement des réseaux d'épidémiosurveillance, car les suspicions des maladies surveillées seront rarement étayées par un diagnostic de laboratoire, ce qui entraînera, sans nul doute, une démotivation des acteurs de terrain. Cette contrainte peut être liée à l'insuffisance des ressources financières, matérielles et/ou humaines. Pour pallier cela, il est nécessaire de développer des outils d'aide à la décision clinique robustes (par exemple, 16).

La représentativité d'un réseau d'épidémiosurveillance est un des critères d'efficacité importants. Idéalement, dans un objectif de lutte ou de détection précoce d'une maladie exotique, le réseau national doit couvrir l'ensemble du pays. Nous constatons cependant que ce n'est pas souvent le cas, pour des raisons économiques et financières. Compte tenu des systèmes d'élevage pratiqués dans les pays enquêtés, généralement de type extensif, ainsi que de la perméabilité des frontières, la représentativité géographique d'un réseau dépasse largement le critère national. Il est donc nécessaire que la représentativité d'un réseau à l'échelle nationale tienne compte de cette caractéristique et soit intégrée dans une perspective régionale. Dans tous les cas, pour améliorer la possibilité de détection précoce des maladies prioritaires, il conviendrait qu'au niveau de chaque poste vétérinaire il y ait un dispositif de surveillance épidémiologique et que les vétérinaires privés participent davantage à celui-ci.

La standardisation de la collecte des données épidémiologiques par les acteurs de terrain est en général assurée dans la plupart des réseaux d'épidémiosurveillance car des formations initiales et des sessions de recyclage sont organisées. Presque tous les réseaux enquêtés utilisent des techniques passives pour la surveillance de la plupart des maladies (6) alors que la surveillance active est réservée à la procédure de l'OIE pour l'obtention du statut de pays indemne de peste bovine. Cette dernière est en réalité une action ponctuelle. Elle peut être assimilée à une enquête transversale reconduite annuellement. Les modalités de cette surveillance active diffèrent d'un pays à l'autre. Certains pays organisent des réunions de sensibilisation des éleveurs et des visites régulières des troupeaux pour la recherche des maladies à surveiller. L'efficacité de ces différentes méthodes demande à être étudiée pour permettre de porter un jugement sur la pertinence de leur mise en œuvre.

Dans les réseaux d'épidémiosurveillance enquêtés, la transmission des données se fait par le biais de l'édition de bulletins épidémiologiques. Dans certains réseaux, les données étaient collectées par des postes de surveillance puis transitaient par des unités régionales avant d'arriver à l'unité centrale. Pour d'autres, le circuit était plus complexe avec un nombre plus important d'intermédiaires. Plus le système est complexe, plus il est probable que l'adhésion des informateurs soit faible (3) et que le temps de transmission des données soit long. Or, la rapidité de transmission des données et de l'information est un indicateur clef de l'efficacité d'un réseau d'épidémiosurveillance car c'est l'un des paramètres essentiels d'une réaction efficace à une épizootie.

La gestion et le traitement des données sont également un point critique très important à maîtriser pour assurer l'efficacité d'un réseau. Ils conditionnent également la transmission et la diffusion des résultats, et donc l'efficacité de la réaction à un événement sanitaire quelconque.

Tous les réseaux d'épidémiosurveillance enquêtés disposent de la base de données PID/ARIS mise en place par le PACE. Mais, dans la plupart des pays, son utilisation semble poser des problèmes. Le PID/ARIS est une base de données conçue dans le cadre de la régionalisation des réseaux. Son objectif est en effet de permettre l'agrégation des données à l'échelon régional mais il apparaît cependant que son développement reste insuffisant pour une utilisation optimale à l'échelon national, essentiellement en raison d'un manque de flexibilité qui ne permet pas son adaptation aux besoins spécifiques des pays. Un réseau 
d'épidémiosurveillance est en effet appelé à évoluer, avec des possibilités de changement dans les protocoles de surveillance, des modifications de fiches de collecte de données ou l'introduction d'une nouvelle maladie. Tous ces changements entraînent parfois la nécessité de modifier de manière plus ou moins approfondie la base de données utilisée. Pour ce faire, celle-ci doit être simple, flexible et permettre de répondre aisément aux modifications éventuelles souhaitées. Une base de données écrite à l'aide du logiciel Access $^{\circledR}$ est une solution possible pour les réseaux nationaux, car elle répond facilement aux besoins de flexibilité.

La diffusion des informations produites par le réseau d'épidémiosurveillance est un élément déterminant de motivation des acteurs de terrain et permet de diffuser à l'extérieur des preuves de son bon fonctionnement. Le bulletin d'information épidémiologique est l'outil le mieux adapté pour la diffusion de l'information tant au niveau national qu'international. Il est le signe de l'existence et de l'opérationnalité du réseau. Sa périodicité doit être respectée. On peut cependant constater que dans le cadre de cette enquête, la majorité des pays ont du mal à respecter cette nécessité.

L'évaluation interne des réseaux d'épidémiosurveillance est indispensable pour en assurer la gestion continue (13). Cette étude a montré que tous les réseaux d'épidémiosurveillance enquêtés disposent d'indicateurs de performance pour leur évaluation. Cependant, ils sont rarement utilisés. Ces indicateurs permettent d'identifier et de mesurer, en temps réel, les points de dysfonctionnement du réseau afin d'apporter les corrections qui s'imposent. Par conséquent, ils doivent être régulièrement utilisés et mis à jour. Il ne sert à rien d'élaborer des indicateurs et de ne pas les utiliser.

\section{Conclusion}

Cette enquête a permis de confirmer l'existence de réseaux d'épidémiosurveillance bien formalisés et structurés dans neuf pays d'Afrique francophone de l'Ouest et du Centre. Cependant, les résultats de l'enquête indiquent la nécessité d'améliorer l'efficacité de certains éléments des réseaux d'épidémiosurveillance, en particulier, dans le domaine de la collecte des données. Essentiellement centrée sur les structures et les activités mises en place par les réseaux d'épidémiosurveillance, cette enquête n'a toutefois pas permis d'aborder de manière approfondie l'efficacité de ces réseaux. Il conviendrait donc de réaliser en complément une étude de leurs résultats.

La similitude manifeste des réseaux d'épidémiosurveillance enquêtés est un atout majeur. Elle permet en effet de faciliter l'harmonisation régionale des procédures de surveillance ainsi que le développement d'outils d'évaluation et de suivi des réseaux à l'échelon régional.

On peut enfin constater que les États africains ont réalisé un effort important pour mettre en place des réseaux d'épidémiosurveillance qui contribuent fortement au renforcement des Services vétérinaires. Il est désormais essentiel que ces mêmes États puissent être en mesure de pérenniser les réseaux d'épidémiosurveillance existants en mobilisant les ressources tant matérielles qu'humaines et financières indispensables à leur fonctionnement. Il serait en effet dommageable pour la crédibilité des Services vétérinaires de laisser disparaître ces précieux outils de prévention et d'aide à la décision qui sont en place et dont il ne reste plus qu'à assurer et améliorer le fonctionnement.

\section{Remerciements}

Les animateurs des réseaux de surveillance des maladies animales d'Afrique de l'Ouest et du Centre sont vivement remerciés pour leur participation à l'enquête. Celle-ci a été financée par la Direction générale pour la coopération au développement (DGCD). 


\title{
Epidemiological surveillance networks for animal diseases in French-speaking West and Central Africa
}

\author{
M. Ouagal, P. Hendrikx, D. Berkvens, A. Ncharé, B. Cissé, P.Y. Akpeli, \\ K. Sory \& C. Saegerman
}

\section{Summary}

One of the objectives of the Pan African Programme for the Control of Epizootics (PACE) was to set up epidemiological surveillance networks in African countries. A survey based on a written questionnaire was conducted to review the technical and institutional organisation of epidemiological surveillance networks in nine French-speaking countries, including five in West Africa (Senegal, Burkina Faso, Côte d'Ivoire, Togo and Guinea) and four in Central Africa (Cameroon, Central African Republic, Democratic Republic of Congo and Chad). The survey results showed that there are more similarities than differences among epidemiological surveillance networks. In general, they were found to be technically and institutionally well established. However, the two weaknesses of the majority of networks are the inadequate diagnostic capacity of laboratories and the insufficient operationality of steering committees. Epidemiological surveillance should exclusively be the domain of Veterinary Services and it is crucial for ensuring that any change in the health status of an animal population is detected rapidly. However, the networks' continuing survival after external financing ceases is generally not guaranteed because, in many cases, governments fail to fund them adequately.

\section{Keywords}

Animal disease - Central Africa - Domestic animal - Epidemiological surveillance Epidemiological surveillance network - Laboratory - Pan African Programme for the Control of Epizootics (PACE) - Veterinary Services - West Africa.

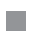

\section{Las redes de vigilancia epidemiológica zoosanitaria en los países francófonos del África Occidental y Central}

\author{
M. Ouagal, P. Hendrikx, D. Berkvens, A. Ncharé, B. Cissé, P.Y. Akpeli, \\ K. Sory \& C. Saegerman
}

\section{Resumen}

Uno de los objetivos del Programa Panafricano de Control de Epizootias (PACE) era el de poner en marcha redes de vigilancia epidemiológica en los países del continente. Los autores describen una encuesta destinada a hacer balance de la situación organizativa de esas redes desde el punto de vista técnico e institucional. Para ello se distribuyó un cuestionario escrito en nueve países francófonos, cinco de África Occidental (Senegal, Burkina Faso, Côte d'Ivoire, Togo y Guinea Conakry) y cuatro de África Central (Camerún, República Centroafricana, República Democrática del Congo y Chad). Los resultados de dicha encuesta ponen de manifiesto que entre todas esas redes existen más semejanzas que divergencias. En términos generales están bien formalizadas tanto técnica como institucionalmente. Sin embargo, la mayoría 
de ellas presentan dos puntos débiles: la insuficiente capacidad de diagnóstico de los laboratorios y la escasa operatividad de los comités de seguimiento. La vigilancia epidemiológica es una actividad fundamental para detectar rápidamente cualquier modificación del estado sanitario de una población animal, por lo que debería ser una labor intrínseca de los Servicios Veterinarios. Pese a ello, la continuidad a largo plazo de las redes no está en general garantizada cuando la financiación exterior llega a su término, pues suele ocurrir que los Estados no se hagan cargo de ellas de forma conveniente.

\section{Palabras clave}

África Central - África Occidental - Animal doméstico - Enfermedad animal Laboratorio - Programa Panafricano de Control de Epizootias (PACE) - Red de vigilancia epidemiológica - Servicios Veterinarios - Vigilancia epidemiológica.

\section{Références}

1. Cardinale E. \& Hendrikx P. (2000). - The Senegalese epidemiosurveillance network on poultry diseases (RESESAV). In International Symposium on Veterinary Epidemiology and Economics, Breckenridge, Colorado, USA. International Society for Veterinary Epidemiology and Economics.

2. Caribbean Animal Health Network (2007). - Surveillance networks. Page web: http://www.caribvet.net/surveillance. php (consultée le 20 décembre 2007).

3. Dabis F, Drücker J. \& Moren A. (1992). - Évaluer un système de surveillance. In Épidémiologie d'intervention (F. Dabis, J. Drücker \& A. Moren, édit.). Arnette, Paris, 109-141.

4. Dufour B. (1995). - Les réseaux français d'épidémiosurveillance animale. Épidémiol. Santé anim., 27, 1-10.

5. Dufour B., Hendrikx P. \& Toma B. (2006). - Élaboration et mise en place de systèmes de surveillance épidémiologique des maladies à haut risque dans les pays développés. Rev. sci. tech. Off. int. Epiz., 25 (1), 187-198.

6. Dufour B. \& Hendrikx P. (2007). - La surveillance épidémiologique en santé animale, $2^{e}$ éd. A.E.E.M.A et Quae, Paris.

7. Hendrikx P., Bidjeh K., Ganda K., Ouagal M., Haggar A.I., Saboun M., Maho A. \& Idriss A. (1997). - Le réseau d'épidémiosurveillance des maladies animales au Tchad. Rev. sci. tech. Off. int. Epiz., 16 (3), 759-769.
8. Hendrikx P. \& Dufour B. (2004). - Méthode d'élaboration des indicateurs de performance des réseaux de surveillance épidémiologique des maladies animales. Épidémiol. Santé anim., 46, 71-85.

9. James A.D. (1998). - Guide pour la surveillance épidémiologique de la peste bovine. Rev. sci. tech. Off. int. Epiz., 17 (3), 810-824.

10. Organisation mondiale de la santé animale (OIE) (2006). Liste des maladies de l'OIE en vigueur en 2006. Page web: http://www.oie.int/fr/maladies/fr_classification2006.htm (consultée le 20 décembre 2007).

11. Organisation mondiale de la santé animale (OIE) (2006). Acte du séminaire régional OIE/UA-IBAR/FAO. Politiques de santé animale, évaluation des services vétérinaires et rôle des éleveurs dans la surveillance des maladies animales. 13-15 février 2006, N’Djamena, Tchad, 440 pp. Page web: http://www.oie.int/downld/NDJAMENA.pdf (consultée le 13 octobre 2008).

12. Organisation mondiale de la santé animale (OIE) (2007). Code sanitaire pour les animaux terrestres. Page web: http://www.oie.int/fr/normes/fr_mcode.htm?eldlo (consultée le 20 décembre 2007).

13. Ouagal M., Berkvens D. \& Hendrikx P. (2004). - Élaboration d'indicateurs de performance du fonctionnement du réseau tchadien d'épidémiosurveillance des maladies animales: le REPIMAT. Épidémiol. Santé anim., 45, 101-112. 
14. Paskin R. (1999). - Manual on livestock disease surveillance and information systems. Organisation des Nations unies pour l'agriculture et l'alimentation (FAO), Rome, $71 \mathrm{pp}$.

15. Saegerman C., Dechamps P., Roels S., Petroff K., Geeroms R., Torck G., Dufey J., Fourez R., Hamelryckx M., Cormann A., Viatour P., De Coninck V., Lomba F., Vermeersch J.-P., Hallet I., Lhost O., Leemans M., Vandersanden A., Peharpre D., Brochier B., Costy F., Pastoret P.-P., Thiry E. \& Vanopdenbosch E. (2002). - Épidémiosurveillance de l'encéphalopathie spongiforme bovine en Belgique : bilan de l’année 1999. Ann. Méd. vét., 145, 47-58.

16. Saegerman C., Speybroeck N., Roels S., Vanopdenbosch E., Thiry E. \& Berkvens D. (2004). - Decision support tools for clinical diagnosis of diseases in cows with suspected bovine spongiform encephalopathy. J. clin. Microbiol., 42, 172-178.

17. Salman M.D., Stärk K.D.C. \& Zepeda C. (2003). - Quality assurance applied to animal disease surveillance system. Rev. sci. tech. Off. int. Epiz., 22 (3), 689-696.

18. Sidibé A.S. (2003). - Les apports de l'assurance qualité à une organisation nationale vétérinaire dans les pays en développement: le cas de l'Afrique. Rev. sci. tech. Off. int. Epiz., 22 (3), 679-688.
19. Squarzoni C., Bendali F., Denormandie N., Bastiaensen P. \& Diop B. (2005). - Les réseaux d'épidémio-surveillance dans treize pays d'Afrique de l'Ouest du PACE : état des lieux et évaluation de leur fonctionnement en 2004. Épidémiol. Santé anim., 48, 69-80.

20. Tambi E.N., Maina O.W. \& Mariner J.C. (2004). - Ex-ante economic analysis of animal disease surveillance. Rev. sci. tech. Off. int. Epiz., 23 (3), 737-752.

21. Tambi N.E., Maina W.O. \& Ndi C. (2006). - An estimation of the economic impact of contagious bovine pleuropneumonia in Africa. Rev. sci. tech. Off. int. Epiz., 25 (3), 999-1011.

22. Toma B., Dufour B., Sanaa M., Bénet J.J., Shaw A., Moutou F. \& Louzà A. (2001). - Épidémiologie appliquée à la lutte collective contre les maladies animales transmissibles majeures, $2^{e}$ éd. Association pour l'étude de l'épidémiologie des maladies animales, Maisons-Alfort, $732 \mathrm{pp}$. 